\title{
IMPLICACIONES DE LA CIUDADANÍA PLENA: TODOS LOS CIUDADANOS CON LOS MISMOS DERECHOS. EL CASO DEL MATRIMONIO IGUALITARIO
}

Jairo Enrique Ordóñez G. ${ }^{1}$

\section{Resumen}

Esta investigación analiza el cubrimiento periodístico del matrimonio y la adopción por parte de parejas del mismo sexo en cinco medios de comunicación nacionales de amplia circulación. Se evidencia que los medios seleccionados toman una posición neutral sobre la adopción por parte de las parejas del mismo sexo, mayoritariamente una posición a favor del matrimonio igualitario y que tienen una orientación elitista y religiosa.

Palabras clave: Matrimonio igualitario, adopción, parejas del mismo sexo, medios de comunicación, Colombia.

1 Comunicador social con doctorado en Psicología Social de la Universidad de Konstanz, Alemania. Actualmente es profesor de la maestría en Comunicación de la Universidad Santo Tomás, sede Bogotá. Contacto: jairo_e_20oo@yahoo.com 


\section{Introducción}

Dados los importantes cambios que se están dando en la conformación de las familias y que implican concebir y aceptar nuevos modelos de parejas, matrimonios, paternidad, maternidad y roles de género, entre otros; se hace indispensable analizar el papel que los medios de comunicación juegan al cubrir las noticias alrededor del matrimonio y la adopción por parte de parejas del mismo sexo, ya que podrían estar contribuyendo a reproducir prejuicios o, por el contrario, podrían representar una oportunidad para crear una sociedad más incluyente y diversa.

En el contexto colombiano, está teniendo lugar un desarrollo jurídico trascendental para las parejas del mismo sexo. Cabe mencionar aquí la equiparación de las parejas heterosexuales y las del mismo sexo, que estipula que si el Congreso de la República no legisla al respecto, se homologan los derechos y obligaciones del matrimonio existente a ambos tipos de pareja: «Si el 20 de junio de 2013 el Congreso de la República no ha expedido la legislación correspondiente, las parejas del mismo sexo podrán acudir ante notario o juez competente a formalizar y solemnizar su vínculo contractual» (Corte Constitucional, Sentencia $\mathrm{C}_{577 / 11)}$. Esta sentencia tiene el fin de que en Colombia las parejas del mismo sexo no sean discriminadas o tengan déficit de protección por parte del Estado. Por ello, la Corte Constitucional exhortó:

(..) al Congreso de la República para que antes del 20 de junio de 2013 legisle, de manera sistemática y organizada, sobre los derechos de las parejas del mismo sexo con la finalidad de eliminar el déficit de protección que, según los términos de esta sentencia, afecta a las mencionadas parejas (Corte Constitucional. Sentencia C577/11).

De igual manera, la Corte Constitucional, al fallar un caso que permitió la adopción a una pareja del mismo sexo, con la condición de que el menor de edad fuera hijo biológico de una de las conyuges, sentó un precedente hacia la adopción igualitaria. Actualmente cursan otras demandas que podrían hacer que la adopción no tuviera condicionamientos, como los lazos biológicos, y que se diera en los mismos términos y condiciones en que se concede a las personas o parejas heterosexuales.

Ambos procesos, el matrimonio y la adopción igualitarios, están íntimamente ligados, ya que todo matrimonio, o su equivalente, forman una familia, y en la gran mayoría de familias hay hijos o el deseo de tenerlos. Por tal motivo, es muy probable que en el corto plazo 
experimentemos una gran transformación jurídica en nuestro país, tal y como lo han hecho varias naciones en Europa, Norteamérica, África y América Latina. No obstante, el hecho de que la sentencia de la Corte Constitucional C577/11 no haya nombrado directamente la palabra matrimonio abrió un espacio para interpretaciones y por ese motivo los notarios se han abstenido de realizar matrimonios igualitarios, atendiendo a directivas de sus superiores, mientras que algunos jueces han accedido a realizar uniones entre parejas del mismo sexo, equiparables en todo sentido a un matrimonio civil heterosexual, y otros se han rehusado.

Actualmente esta ambigüedad en la jurisprudencia ha hecho que pocas personas quieran acceder al matrimonio igualitario, pues no hay estabilidad jurídica, mientras se deciden varias demandas interpuestas por diversas personas y organizaciones. Todo parece indicar que el tema retornará nuevamente a la Corte Constitucional para que aclare y precise su sentencia, lo cual puede tardar varios años. No obstante, quienes se han casado, aunque los jueces usan el mismo término de la Corte: solemnizar, legalmente cuentan con todos los derechos y deberes de un matrimonio civil heterosexual, incluyendo, en teoría, la adopción, pues pertenecer a la comunidad LGBTI no es impedimento para adoptar, aunque en la práctica a estas personas sí se les niega ese derecho, aduciendo diferentes argumentos legales y morales. Por lo anterior, es muy importante que la Corte Constitucional se pronuncié de fondo sobre la adopción igualitaria, ya que en la actualidad solo se permite si el menor tiene un vínculo biológico con alguno de los cónyuges, lo cual aplica para muy pocos casos. En aras de la igualdad y la equidad se requiere que las parejas del mismo sexo puedan adoptar bajo las mismas condiciones de sus pares heterosexuales.

Idealmente, estas transformaciones jurídicas deben estar acompañadas de cambios de imaginarios y de actitudes para que se conviertan en hechos plenos y no solamente en documentos, como pasa con muchas leyes y normas en Colombia. Es en este proceso transformador donde los medios tienen un papel clave para que el ciudadano del común pueda ver que dichos cambios se sustentan en la democracia y en el precepto de que todos los ciudadanos tienen los mismos derechos, sin distinción de raza, credo, condición social u orientación sexual, como debe serlo en cualquier estado de derecho.

De ahí la importancia de observary monitorear a influyentes medios de comunicación y oportunidades de mejora para que se potencialicen como agentes de inclusión, democracia, ciudadanía y paz.

El presente trabajo se inscribe en la línea de las reivindicaciones sociales que iniciaron en los años 5o, cuando se empezó a hablar de combatir la discriminación contra 
los afroamericanos. Estas siguieron su camino en los años 6o, con el reclamo de las mujeres por la igualdad de derechos y, finalmente, a partir de los 8o, con la inclusión de todas las minorías sexuales. Todas estas reivindicaciones buscan la consolidación de las denominadas ciudadanías plenas: igualdad de derechos para todos los ciudadanos.

\section{Objetivos de la investigación}

- Analizar el cubrimiento que hacen importantes medios colombianos de las noticias alrededor del matrimonio y la adopción por parte de parejas del mismo sexo.

- Describir las implicaciones de dicho cubrimiento en una sociedad democrática.

- Hacer explícitas las posiciones a favor y en contra de cada medio sobre estas cuestiones.

- Analizar si existe una orientación religiosa y elitista en el discurso mediático.

\section{Conceptos clave}

Medios de comunicación como la televisión, la prensa, Internet y las redes sociales han redefinido la interacción social de millones de personas y han creado nuevas posibilidades de veeduría pública, gobierno, trabajo e, incluso, protesta social, entre otros; sin embargo, también han reproducido representaciones que propician la propagación de estereotipos y de distintas formas de violencia simbólica.

Para hacer un estudio de este fenómeno, es necesario definir, en este marco conceptual, seis términos: Enfoque diferencial (el enfoque de nuestro proyecto); perspectiva de género (un concepto esencial en este trabajo); ciudadanía (en lo que se quiere incidir); estereotipo (la principal categoría a medir y de la que se desprenderán varias subcategorías); orientación elitista, y discriminación.

Enfoque diferencial: Es un concepto relacionado con el derecho a la igualdad. Implica que los ciudadanos en situaciones similares deben ser tratados de manera igual y que aquellos en situaciones distintas deben tratarse de forma distinta, proporcionalmente a dicha diferencia. (Ministerio de Cultura, 2009).

Perspectiva de género: Es uno de los conceptos teóricos más importantes que ha construido el feminismo y que hoy en día se hace extensivo a las minorías sexuales. 
Establece cómo la sociedad ha estructurado un conjunto de normas que favorecen la heterosexualidad y la dominación masculina, que en muchas ocasiones es fuente de conflicto social (Rubin, 1975). Asimismo, establece que hay otras posibilidades frente a esta estructura y por eso se interesa en las mujeres y en todas las posibles identidades sexuales que se distancian de la heteronormatividad masculina predominante, y que para efectos prácticos muchas veces se agrupan en la sigla LGBTI.

Ciudadanía: Es un concepto conflictivo ya que está asociado a la hegemonía heterosexual masculina: «La definición liberal de ciudadanía universaliza las características de un sujeto heterosexual masculino que provoca tensiones en el momento de diseñar e implementar políticas públicas que atienden necesidades de diversos grupos interesados en impugnar las categorías sexuales y genéricas hegemónicas» (Moreno, 2006, p. 120). Sin embargo, es una definición que presenta muy bien la tensión que se da cuando las mujeres o la población LGBTI reclaman derechos que les han sido sistemáticamente negados. De ahí que se hable de ciudadanía activa para indicar la necesidad de los ciudadanos marginados de reclamar sus derechos: «La ciudadanía activa supone que la persona está dispuesta a reclamar sus derechos y a ejercerlos» (Cortina, 2006).

Y esa ciudadanía activa encuentra en los medios de comunicación una respuesta a su clamor por ser escuchados y atendidos:

Lo propio de la ciudadanía hoy es hallarse asociada al reconocimiento recíproco; esto es al derecho a informar y ser informado, de hablar y ser escuchado imprescindible para poder participar en las decisiones que conciernen a la colectividad. Una de las formas hoy más flagrantes de exclusión ciudadana se sitúa [...] en la desposesión del derecho a ser visto y oído, que equivale al de existir/contar socialmente, tanto en el terreno individual como colectivo, en el de las mayorías como de las minorías (Winocur, 2002, p. 181).

Estereotipo: Allport (1979) define estereotipo como una creencia exagerada con, o acerca de, las costumbres y atributos de un determinado grupo o categoría social, en función de la cual se justifica o racionaliza nuestra conducta en relación a dicha categoría. De esta categoría se desprenden subcategorías como lenguaje sexista, lenguaje homófobo, lenguaje incluyente, lenguaje elitista, lenguaje pro y de-escalación, entre otros.

Para esta investigación también se han tomado los postulados teóricos de Johan Galtung y Richard Vincent (1992) en lo concerniente a la orientación elitista de los medios, 
el manejo de las fuentes y el protagonismo dado en los artículos periodísticos a ciertas personas y estructuras con poder e influencia política (élite).

Orientación elitista de los medios: Tiene que ver con los protagonistas de las notas periodísticas. Presenta dos líneas principales; la primera se refiere a personas y estructuras élite, que hace referencia a aquellas personas y organizaciones con privilegios, dinero e influencias sociales y políticas, como presidentes, el Congreso, la iglesia católica, etc. La segunda línea es la de personas y estructuras no élite, que se refiere a aquellas sin privilegios, dinero o influencias sociales y políticas, como pueden serlo un estudiante, un líder comunitario de un barrio popular o una ONG de carácter local (Galtung y Vincent, 1992).

Para esta categoría, también han sido importantes los postulados de Gadi Wolfsfeld (1997), quien plantea las relaciones existentes entre los medios y los conflictos políticos como aquellos surgidos a raíz de la discusión y de la aprobación de leyes que implican la reivindicación de derechos civiles para las mayorías (los no élite).

Discriminación y diversidad sexual. La sociedad colombiana tiende a ser poco tolerante con quienes abogan por el derecho a la diferencia, en especial, el derecho a la diversidad sexual, tal y como lo indica un estudio de Colombia Diversa realizado entre 2008 y 2009 :

Cuatro casos de asesinato de activistas y defensores de derechos humanos LGBT tuvieron una especial repercusión en esta comunidad [...]:

- Febrero 16 del 2008. Fue asesinado Fredy Darío Pineda, un líder, defensor y activista de los derechos humanos del sector LGBT.

- Marzo 6 del 2009. Asesinado en Cali Álvaro Miguel Rivera, activista de diferentes organizaciones y proyectos de derechos de la población LGBT y el VIH/ Sida.

- Julio 12 del 2009. Alexander Guao Sierra, reconocido líder de la población LGBT, fue hallado muerto en su residencia. Los indicios del asesinato indicaron violencia extrema.

- Octubre 28 del 2009. Asesinato en Bogotá de Wanda Fox, del proyecto Zona Trans de Procrear en Bogotá, organización que trabaja para hacer visibles los derechos humanos de las transgeneristas (Colombia Diversa, 2009; p. 127). 
Pero no solamente los activistas son víctimas; según la misma organización, casi un centenar de personas fueron asesinadas, muy probablemente, por el simple hecho de tener una identidad de género diferente a la de la mayoría de la población: «A partir de la nueva información se pudo establecer que se habrían perpetrado al menos 32 homicidios más, lo que permite afirmar que entre el 2006 y el 2007 fueron asesinadas al menos 99 personas LGBT» (Colombia Diversa, 2009, p.16). Se ha identificado que de estos casos, 37 fueron crímenes de odio y están por establecer las causas de los demás. En muchas ocasiones es imposible establecer la verdadera causa del asesinato ya que los familiares no admiten la orientación sexual de su ser querido por vergüenza y, en otros casos, las autoridades no investigan por considerar que de alguna forma las víctimas se lo merecían por ser como eran. Como ejemplo podemos ver la actitud de un fiscal en Cartagena:

La Fiscalía, lejos de hacer un análisis sobre los móviles del homicida, se limita a hacer especulaciones prejuiciadas sin ningún fundamento o fin para el objeto de la investigación. Las valoraciones no se hacen con el fin de formular un agravante, sino para justificar al victimario, endilgando la responsabilidad al comportamiento de la víctima y validando el homicidio. (Colombia Diversa, 2009, p. 19).

Esta situación refleja los graves prejuicios sociales que pesan sobre las personas LGBTI; es indispensable educar a los periodistas y audiencias sobre la imperiosa necesidad de aceptar la diferencia y de informar sobre la grave situación de derechos humanos de la comunidad LGBTI como consecuencia de una sociedad intolerante, que resuelve sus conflictos con la aniquilación del otro. El periodismo pro-paz orientado hacia la deescalación puede brindar un gran aporte para que la sociedad censure la violencia y para que los mismos periodistas y audiencias sean conscientes de su rol activo en la construcción de una opinión pública que valore la democracia, la diversidad y la paz.

\section{Método}

Esta investigación es de enfoque cuantitativo y tiene un alcance descriptivo. Para seleccionar la muestra de análisis se hizo un seguimiento a las versiones impresas de cinco medios de circulación nacional y de amplia circulación:

- Revista Semana, la revista más influyente del país, editada en Bogotá. 
- El Tiempo, el periódico más leído y más influyente en Colombia, editado en Bogotá.

- ElEspectador, el diario más antiguo de Colombia, uno de lo más leídos y reconocidos por su trayectoria y prestigio de muchos de sus periodistas, especialmente los que escriben columnas de opinión. Editado en Bogotá.

- $E l$ Espacio, que fue uno de los diarios populares más importantes de Colombia, uno de los más antiguos y leídos (dejó de circular en el 2013). Se editaba en Bogotá.

- El País. El periódico más influyente del sur del país y de amplia circulación en una de las zonas de Colombia con más población. Editado en Cali (Valle del Cauca).

El periodo analizado comprende las publicaciones entre junio de 2010 y diciembre de 2013. El inicio del período de observación corresponde a la fecha en que la Corte Constitucional se pronunció dándole dos años al Congreso para que legisle y garantice a las parejas del mismo sexo los mismos derechos de las parejas heterosexuales. En total se revisaron 3500 ediciones y en ellas se encontraron 255 piezas periodísticas de todo tipo (opinión, noticia, crónica, entrevista, editorial, caricaturas, etc). Por medio de comunicación, el aporte de artículos fue el siguiente:

Tabla 1. Aporte de artículos

\begin{tabular}{lll}
\hline \multicolumn{1}{c}{ Medio } & N. ${ }^{\circ}$ de Art. & $\%$ \\
\hline El Tiempo & 96 & 37,6 \\
\hline El Espectador & 81 & 31,8 \\
\hline El País & 43 & 16,9 \\
\hline El Espacio & 22 & 8,6 \\
\hline Semana & 13 & 5,1 \\
\hline Total & 255 & 100 \\
\hline
\end{tabular}

Fuente: Elaboración propia

Cada una de las piezas halladas fue sistematizada en una matriz, especificando si había una posición a favor, en contra o neutral sobre la adopción y el matrimonio por parte de parejas del mismo sexo. Se examinó el porcentaje de consultas a las siguientes fuentes:

- La iglesia (para medir la orientación religiosa).

- La comunidad LGBTI (los directamente implicados). 
- Representantes del Estado y partidos políticos (los directamente implicados en la elaboración y votación de la ley exigida por la Corte Constitucional).

- Las ONG, con el fin de examinar la consulta de muchos colectivos LGBTI que se encuentran asociados como ONG y medir la consulta a fuentes no tradicionales.

- Ciudadanos del común, para ver si se tienen en cuenta a la hora de informar.

- También se detalló si había presencia de personas y estructuras élite, así como personas y estructuras no élite.

Una vez completada la matriz se realizó un análisis de contenido de todas las piezas recolectadas.

\section{Resultados}

$\mathrm{Al}$ analizar en conjunto los 5 medios seleccionados, se obtuvieron los siguientes hallazgos sobre los temas de adopción y matrimonio:

Tabla 2. Hallazgos sobre adopción y matrimonio

\begin{tabular}{llll}
\hline \multicolumn{1}{c}{ Categoría/ Posición } & Artículos a favor & Artículos en contra & Artículos neutrales \\
\hline Adopción & $32,9 \%$ & $27,1 \%$ & $40,0 \%$ \\
\hline Matrimonio & $48 \%$ & $19,3 \%$ & $32,6 \%$ \\
\hline
\end{tabular}

Fuente: Elaboración propia.

Tabla 3. Hallazgos sobre las fuentes

\begin{tabular}{ll}
\hline \multicolumn{1}{c}{ Fuente } & Artículos que la citan \\
\hline Iglesia & $7,3 \%$ \\
\hline Comunidad LGBTI & $11,5 \%$ \\
\hline Representantes del Estado & $22,6 \%$ \\
\hline Ciudadano del común & $9,4 \%$ \\
\hline Integrante partido político & $5,4 \%$ \\
\hline ONG & $1,8 \%$ \\
\hline
\end{tabular}

Fuente: Elaboración propia. 
Tabla 4. Orientación élite de los artículos

\begin{tabular}{lc}
\hline \multicolumn{1}{c}{ Presencia de... } & Artículos en los que aparecen \\
\hline Personas élite & $20 \%$ \\
\hline Personas no élite & $14,2 \%$ \\
\hline Estructuras élite & $43,5 \%$ \\
\hline Estructuras no élite & $22,4 \%$ \\
\hline
\end{tabular}

Fuente: Elaboración propia.

Por medio de comunicación, se obtuvieron los siguientes resultados:

EL TIEMPO dedicó 96 artículos al tema.

Tabla 5. Hallazgos sobre adopción y matrimonio en $\varepsilon l$ Tiempo

\begin{tabular}{lccc}
\hline \multicolumn{1}{c}{ Categoría/ Posición } & Artículos a favor & Artículos en contra & Artículos neutrales \\
\hline Adopción & $26,1 \%$ & $28,3 \%$ & $45,7 \%$ \\
\hline Matrimonio & $42,7 \%$ & $16,7 \%$ & $40,6 \%$ \\
\hline
\end{tabular}

Fuente: Elaboración propia.

Tabla 6. Resumen sobre los hallazgos sobre las fuentes de El Tiempo

\begin{tabular}{lc}
\hline \multicolumn{1}{c}{ Fuente } & Artículos que la citan \\
\hline Iglesia & $6,3 \%$ \\
\hline Comunidad LGBTI & $11,8 \%$ \\
\hline Representantes del Estado & $17,6 \%$ \\
\hline Ciudadano del común & $11,8 \%$ \\
\hline Integrante partido político & $5,9 \%$ \\
\hline ONG & $3,2 \%$ \\
\hline
\end{tabular}

Fuente: Elaboración propia. 
Tabla 7. Orientación élite de los artículos de $\varepsilon l$ Tiempo

\begin{tabular}{ll}
\hline Presencia de... & Artículos en los que aparecen \\
\hline Personas élite & $18,4 \%$ \\
\hline Personas no élite & $15,4 \%$ \\
\hline Estructuras élite & $41,7 \%$ \\
\hline Estructuras no élite & $24,6 \%$ \\
\hline
\end{tabular}

Fuente: Elaboración propia.

EL ESPECTADOR dedicó 81 artículos al tema.

Tabla 8. Hallazgos sobre adopción y matrimonio en $\varepsilon l$ Espectador

\begin{tabular}{lccc}
\hline \multicolumn{1}{c}{ Categoría/ Posición } & Artículos a favor & Artículos en contra & Artículos neutrales \\
\hline Adopción & $51,4 \%$ & $17,1 \%$ & $31,4 \%$ \\
\hline Matrimonio & $55,6 \%$ & $18,5 \%$ & $25,9 \%$ \\
\hline
\end{tabular}

Fuente: Elaboración propia.

Tabla 9. Hallazgos sobre las fuentes de $\varepsilon l$ Espectador

\begin{tabular}{lc}
\hline \multicolumn{1}{c}{ Fuente } & Artículos que la citan \\
\hline Iglesia & $7,5 \%$ \\
\hline Comunidad LGBTI & $15,4 \%$ \\
\hline Representantes del Estado & $16,4 \%$ \\
\hline Integrante partido político & $2,3 \%$ \\
\hline Ciudadanos del común & $14,5 \%$ \\
\hline ONG & $0,9 \%$ \\
\hline
\end{tabular}

Fuente: Elaboración propia. 
Tabla 10. Orientación élite de los artículos de $\varepsilon l$ Espectador

\begin{tabular}{ll}
\hline \multicolumn{1}{c}{ Presencia de... } & \multicolumn{1}{c}{ Artículos en los que aparecen } \\
\hline Personas élite & $17,9 \%$ \\
\hline Personas no élite & $22,1 \%$ \\
\hline Estructuras élite & $39,1 \%$ \\
\hline Estructuras no élite & $20,9 \%$ \\
\hline
\end{tabular}

Fuente: Elaboración propia.

EL PAÍS dedicó 43 artículos al tema.

Tabla 12. Hallazgos sobre adopción y matrimonio en $\varepsilon l$ País

\begin{tabular}{lccc}
\hline \multicolumn{1}{c}{ Categoría/ Posición } & Artículos a favor & Artículos en contra & Artículos neutrales \\
\hline Adopción & $17,1 \%$ & $20 \%$ & $62,9 \%$ \\
\hline Matrimonio & $34,9 \%$ & $27,9 \%$ & $37,2 \%$ \\
\hline
\end{tabular}

Fuente: Elaboración propia.

Tabla13. Hallazgos sobre las fuentes de $\varepsilon l$ País

\begin{tabular}{lc}
\hline \multicolumn{1}{c}{ Fuente } & Artículos que la citan \\
\hline Iglesia & $8,3 \%$ \\
\hline Comunidad LGBTI & $12,8 \%$ \\
\hline Representantes del Estado & $19,3 \%$ \\
\hline Partidos políticos & $6,4 \%$ \\
\hline Ciudadanos del común & $13,8 \%$ \\
\hline ONG & $1,8 \%$ \\
\hline
\end{tabular}

Fuente: Elaboración propia. 
Tabla 14. Orientación élite de los artículos de $E l$ País

\begin{tabular}{lc}
\hline \multicolumn{1}{c}{ Presencia de... } & Artículos en los que aparecen \\
\hline Personajes élite & $17,5 \%$ \\
\hline Personajes no élite & $17,5 \%$ \\
\hline Estructuras élite & $42.3 \%$ \\
\hline Estructuras no élite & $22,7 \%$ \\
\hline
\end{tabular}

Fuente: Elaboración propia.

El ESPACIO dedicó 22 artículos al tema.

Tabla 15. Hallazgos sobre adopción y matrimonio en $\varepsilon l$ Espacio

\begin{tabular}{lccc}
\hline Categoría/ Posición & Artículos a favor & Artículos en contra & Artículos neutrales \\
\hline Adopción & $20 \%$ & $20 \%$ & $60 \%$ \\
\hline Matrimonio & $45,5 \%$ & $18,2 \%$ & $36,4 \%$ \\
\hline
\end{tabular}

Fuente: Elaboración propia

Tabla 16. Hallazgos sobre las fuentes de $\varepsilon l$ Espacio

\begin{tabular}{lc}
\hline \multicolumn{1}{c}{ Fuente } & Artículos que la citan \\
\hline Iglesia & $8,2 \%$ \\
\hline Comunidad LGBTI & $8,2 \%$ \\
\hline Representantes del Estado & $28,6 \%$ \\
\hline Partidos políticos & $6,1 \%$ \\
\hline Ciudadanos del común & $4,1 \%$ \\
\hline ONG & $0 \%$ \\
\hline
\end{tabular}

Fuente: Elaboración propia. 
Tabla17. Orientación élite de los artículos de El Espacio

\begin{tabular}{lc}
\hline \multicolumn{1}{c}{ Presencia de... } & Artículos en los que aparecen \\
\hline Personajes élite & $17,4 \%$ \\
\hline Personajes no élite & $8,7 \%$ \\
\hline Estructuras élite & $47,8 \%$ \\
\hline Estructuras no élite & $26,1 \%$ \\
\hline
\end{tabular}

Fuente: Elaboración propia.

REVISTA SEMANA dedicó 13 artículos al tema.

Tabla 18. Hallazgos sobre adopción y matrimonio en Revista Semana

\begin{tabular}{lccc}
\hline Categoría/ Posición & Artículos a favor & Artículos en contra & Artículos neutrales \\
\hline Adopción & $50 \%$ & $50 \%$ & $0 \%$ \\
\hline Matrimonio & $61,5 \%$ & $15,4 \%$ & $23,1 \%$ \\
\hline
\end{tabular}

Fuente: Elaboración propia

Tabla 19. Hallazgos sobre las fuentes de Revista Semana

\begin{tabular}{lc}
\hline \multicolumn{1}{c}{ Fuente } & Artículos que la citan \\
\hline Iglesia & $6,3 \%$ \\
\hline Comunidad LGBTI & $9,4 \%$ \\
\hline Representantes del Estado & $31,3 \%$ \\
\hline Partidos Políticos & $6,3 \%$ \\
\hline Ciudadanos del Común & $3,1 \%$ \\
\hline ONG & $3,1 \%$ \\
\hline
\end{tabular}

Fuente: Elaboración propia. 
Tabla 20. Orientación élite de los artículos de la Revista Semana

\begin{tabular}{lc}
\hline \multicolumn{1}{c}{ Presencia de... } & Artículos en los que aparecen \\
\hline Personas élite & $28,6 \%$ \\
\hline Personas no élite & $7,1 \%$ \\
\hline Estructuras élite & $46,4 \%$ \\
\hline Estructuras no élite & $17,9 \%$ \\
\hline
\end{tabular}

Fuente: Elaboración propia.

\section{Conclusiones}

Cuando se analizan todos los medios en conjunto, predomina una posición neutral frente a la adopción por parte de las parejas del mismo sexo y una posición a favor del matrimonio igualitario. En cuanto a las fuentes, la comunidad LGBTI, que es la directamente afectada, no es la más consultada, aunque es la segunda más importante. La fuente más citada son los representantes del Estado, mientras que la iglesia aparece en un cuarto lugar, apenas por debajo del ciudadano del común, lo que indica que para los medios de comunicación los ciudadanos son tan importantes como la iglesia católica, que prácticamente es la única que se cita y se excluyen a otras con posiciones más liberales como la anglicana y la luterana.

\section{Adopción}

- El único medio que expresa una clara posición a favor de la adopción por parte de parejas del mismo sexo es El Espectador.

- El medio que expresa una mayor tendencia en contra de la adopción por parte de las parejas del mismo sexo es $E l$ Tiempo, aunque en todos los medios predomina una posición neutral o ambigüa sobre el tema -en el caso de la Revista Semana, por ejemplo, un $50 \%$ de los artículos sobre el tema están en contra y un $50 \%$ a favor-.

- El único medio que no presenta artículos neutrales sobre la adopción es la Revista Semana. 


\section{Matrimonio Igualitario}

- El Tiempo tiene una posición ambigüa, aunque con tendencia a favor, sobre el matrimonio igualitario, ya que los porcentajes de la posición a favor y neutral son prácticamente los mismos. La posición en contra es minoritaria.

- El Espectador y la Revista Semana presentan la más clara posición a favor del matrimonio igualitario, siendo la segunda tendencia más importante la neutral, y la minoritaria, la posición en contra.

\section{Fuentes}

- Todos los medios consultan a la iglesia, lo que le da una mirada moral a un tema jurídico.

- La fuente más importante en todos los medios, excepto de $\varepsilon l$ Espectador, son los representantes del Estado, nunca lo es la comunidad LGBTI que es la directamente afectada por los temas tratados.

- En El Espectador las fuentes más importantes, con un porcentaje similar de presencia, son los representantes del Estado y la Comunidad LGBTI.

- En El País, la segunda fuente más consultada es el ciudadano del común.

- En $E l$ Espacio se consulta por igual a la iglesia y a la comunidad LGBTI (constituyen las segundas fuentes más consultadas).

- En El Espectador y la Revista Semana la segunda fuente más consultada es la comunidad LGBTI.

- En El Tiempo, la segunda fuente más consultada, con igual porcentaje, es la comunidad LGBTI y el ciudadano del común.

- En la Revista Semana, los partidos políticos y la iglesia se consultan por igual.

- La fuente menos consultada son las ONG, organizaciones que para este estudio corresponden a colectivos independientes que abogan por los derechos humanos y la comunidad LGBTI. 


\section{Orientación elitista}

- En todos los medios predomina una orientación hacia estructuras élite.

- En todos los medios predomina una orientación hacia personas élite, excepto en $\varepsilon l$ Espectador donde la orientación hacia personas no élite es la predominante.

- En El País la orientación hacia personas élite y hacia personas no élite presenta el mismo porcentaje de presencia.

- El medio con mayor orientación elitista es la Revista Semana, presentando las mayores diferencias entre élite y no élite.

\section{Discusión}

A pesar de su ambigüedad, El Tiempo presenta tendencias a favor del matrimonio igualitario y en contra de la adopción, al publicar más artículos en contra que a favor de la adopción, aunque predomine la posición neutral frente al tema. No obstante, es el medio que más artículos dedica a los temas seleccionados en esta investigación y prioriza a la comunidad LGBTI y al ciudadano del común como fuentes, aunque predomine una orientación elitista. Llama la atención que pese a sus vínculos con empresarios y miembros del gobierno de orientación conservadora, tenga una tendencia «moderada» hacia la construcción de ciudadanía plena, la cual debe reforzarse y hacerse más explícita con el fin de lograr mayor incidencia en la opinión pública.

El único medio que presenta una posición a favor, tanto del matrimonio igualitario como de la adopción, es $\varepsilon l$ Espectador, lo que concuerda con su orientación liberal y el hecho de ser un diario que brinda espacio a informes periodísticos y columnas de opinión de voces críticas, disidentes y de vanguardia. Esta posición a favor de los derechos por los que tanto ha luchado la comunidad LGBTI, tanto en el plano nacional como internacional, concuerda con que $\varepsilon l$ Espectador tenga como una de sus principales fuentes a este colectivo, lo que a su vez explica que este diario sea el único en el que predomina un enfoque hacia las personas no élite.

El País, al igual que $\varepsilon l$ Tiempo, presenta una tendencia neutral sobre la adopción, aunque con más artículos en contra que a favor de la adopción. En cuanto al matrimonio igualitario, predomina la posición neutral, aunque hay más artículos a favor que en contra. Este diario es el que más consulta a la iglesia como fuente, lo que podría indicar que en 
otras regiones la influencia de la iglesia es mayor que en los medios editados en Bogotá. $\varepsilon l$ País se enfoca más en estructuras élite, aunque da igual espacio a personas elite y no elite. El cubrimiento de este diario corresponde en buena medida a lo que se puede esperar de un diario regional y de tendencias conservadoras.

$\varepsilon l$ Espacio es el único diario que no consulta a las ONG. Este diario presenta una clara posición a favor del matrimonio igualitario, pero respecto a la adopción su posición es ambigüa, ya que aunque predomina la neutralidad en este tema, las publicaciones a favor y en contra tienen un mismo 20 \% de representación. $E l$ Espacio tiene una clara orientación elitista, a pesar de autoproclamarse y ser reconocido como un diario de carácter popular. Asimismo, tiene una fuerte orientación religiosa ya que consulta en igual medida a la comunidad LGBTI y a la iglesia.

La Revista Semana, conocida por ser un medio de comunicación que profundiza, con un gran sentido crítico, que se interesa por ejercer veeduría ciudadana sacando a la luz casos de corrupción y de tráfico de influencias, y que además cuenta con una plantilla de notables periodistas como María Jimena Duzán, Daniel Coronel, Antonio Caballero, León Valencia y otros, parece polarizada en torno a la adopción por parte de las parejas del mismo sexo: no hay una posición que predomine y no hay artículos neutrales. Es llamativo que la revista sea progresista en unos temas (control de lo público) y muy conservadora a la hora de defender este derecho civil que contribuye a la ciudadanía plena de la comunidad LGBTI. Sin embargo, tiene una clara posición a favor del matrimonio igualitario.

De otro lado, la Revista Semana es el medio de mayor orientación elitista y el que consulta menos al ciudadano. Esta es una situación preocupante dado que Semana es un gran influenciador de opinión pública en empresarios, estudiantes, profesores e intelectuales; al excluir al ciudadano del común, la Revista Semana está contradiciendo su labor de veeduría ciudadana. En cuanto a las fuentes que consulta, la revista es, junto con El Tiempo, el medio que menos consulta a la iglesia y el que más consulta a las ONG.

Aunque Semana es un medio reconocido por su seriedad, exhibe fuertes tendencias elitistas y es ambiguo frente a la concesión de un derecho a una comunidad vulnerada, contribuyendo así a la llamada existencia de los ciudadanos de «segunda clase», aquellos que tienen todas las obligaciones como ciudadanos, pero no todos los derechos.

Aunque el matrimonio igualitario y la adopción por parte de parejas del mismo sexo no son temas religiosos, sino de orden jurídico, que deben ser analizados por la Corte Constitucional y regulados por el Congreso de la República, la iglesia católica constituye una fuente importante para la mayoría de los medios analizados. Desde el punto de vista 
de la religión, el matrimonio es un ritual; sin embargo, la discusión sobre el matrimonio igualitario y la adopción por parte de las parejas del mismo sexo tiene que ver es con leyes y pronunciamientos jurídicos que pretenden otorgarles a todos los ciudadanos los mismos deberes y derechos civiles, independientemente de su orientación sexual, religión, raza, etc., pues se trata de un tema de derechos ciudadanos y no de moral. Esta última es vista en los medios exclusivamente desde el punto de vista católico, ignorando que muchas religiones, como la luterana, permiten el matrimonio igualitario e incluso algunos de sus jerarcas pertenecen, abiertamente, a la comunidad LGBTI. En este sentido, es mucho lo que hay que indagar para que los medios puedan dar un enfoque al tema desde las libertades civiles y apoyen la edificación de la ciudadanía plena como los constructores de opinión pública que son.

En general, en los medios de comunicación seleccionados predomina un enfoque elitista, en el que los miembros de la élite son los protagonistas del artículo periodístico. Por ello es necesario abogar por darle mayor voz al ciudadano, para saber lo que piensa sobre los temas analizados y conocer si en la opinión pública predominan los estereotipos, la aceptación o cualquier otra tendencia. Este conocimiento sería esencial para diseñar manuales para periodistas y políticas que aboguen por la ciudadanía plena y la no discriminación, teniendo en cuenta que los derechos de las minorías no son sujetos de referendo, lo que implica que si no hay aceptación de ellas, el Estado debe generar una cultura de educación que permita la aceptación y la convivencia pacífica.

No obstante, sorprende que en los medios seleccionados exista una tendencia mayoritaria a favor del matrimonio igualitario; esto contrasta fuertemente con la negativa, en reiteradas ocasiones, del Congreso de la República, en posiciones ampliamente difundidas, ya que son justamente los legisladores los que deben abogar por la ciudadanía plena, independientemente de si la mayoría está de acuerdo, pues, como se mencionó anteriormente, los derechos de las minorías no se definen por referendo ni por voto popular, ya que siempre estarían en desventaja, justamente, por su carácter de minoría. 


\section{Referencias}

Allport, G. (1979). The Nature of Prejudice. Cambrige: Perseus Books

Colombia Diversa. (2009). Todos los deberes, pocos los derechos. Situación de derechos humanos de lesbianas, gay, bisexuales y transgeneristas en Colombia 2008 - 2009. Bogotá: Autor.

Cortina A. (2006). Educación en valores y ciudadanía. En Martínez, M. y Hoyos, G. (Eds.). La formación en valores en sociedades democráticas. Barcelona: Octaedro OEI

Corte Constitucional de Colombia (2011). Sentencia C577/11. Bogotá: Autor.

Galtung, J. y Vincent R.(1992). Global Glasnost: Toward a New World Information and Communication Order? Nueva Jersey: Hampton Press.

Ministerio de Cultura. (2009). Enfoque diferencial y acción sin daño. Bogotá: Autor. Recuperado de: www.mincultura.gov.co/index.php?idcategoria=26023

Moreno, A. (2006). Ciudadanía y sexualidad en la ciudad de Buenos Aires. En Revista Nómadas, (24). Bogotá: Fundación Universidad Central.

Rubin, G. (1975). El tráfico de mujeres: Notas sobre la «economía política» del sexo. En Nueva Antropología, (30). México.

Winocur, R. (2011). Los ciudadanos recreados por los medios. En Callegari, N., y Lizarralde, A., (Comp.). El poder de la comunicación: ¿Urdiendo hilos de paz o de conflicto? (p.p. 179-203). Bogotá: Departamento de Publicaciones USTA.

Wolfsfeld, G. (1997). Media and Political Conflict: News from the Middle East. Cambridge: University Press. 